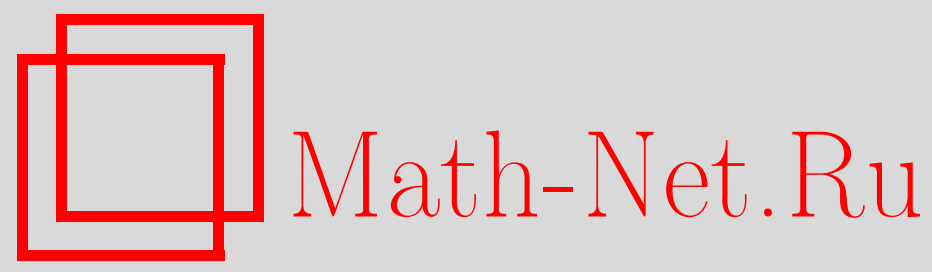

А. А. Вороненко, Об универсальных частичных функциях для класса линейных функций, Дискрет. матем., 2012, том 24, выпуск 3, 62-65

DOI: https://doi.org/10.4213/dm1197

Использование Общероссийского математического портала Math-Net.Ru подразумевает, что вы прочитали и согласны с пользовательским соглашением http://www . mathnet.ru/rus/agreement

Параметры загрузки:

IP : 3.85 .5 .30

26 апреля 2023 г., 14:10:09 


\title{
Об универсальных частичных функциях для класса линейных функций
}

\author{
(C) 2012 г. А. А. Вороненко
}

\begin{abstract}
Рассматривается задача о возможности частичного задания булевой функции таким образом, чтобы предъявление некоторых ее наборов однозначно задавало любую линейную функцию. Показано, что требуемое число наборов области определения растет линейным образом относительно числа переменных.

Работа выполнена при поддержке программы Президента Российской Федерации для поддержки молодых российских ученых - докторов наук, грант МД-757.2011.9.
\end{abstract}

В соответствии с определением из [1] будем называть линейной функцию, представимую в виде $\alpha_{0}+\alpha_{1} x_{1}+\ldots+\alpha_{n} x_{n}$. Назовем не всюду определенную (далее частичную) булеву функцию $f$ от $n$ переменных универсальной, если для любой линейной функции $g$ тех же переменных существует $n+1$ набор из области определения $f$ такой, что единственной линейной функцией, совпадающей с $f$ на этих наборах, является $g$.

Обозначим через $\psi\left(x_{1}, x_{2}, x_{3}, x_{4}\right)$ функцию, равную нулю на нулевом наборе и всех наборах веса 3, единице на всех наборах веса 1 и наборах веса 2, $\psi$ не определена на наборе из всех единиц.

Лемма 1. Функичия $\psi\left(x_{1}, x_{2}, x_{3}, x_{4}\right)$ является универсальной функиией четырех переменHblx.

Доказательство. В силу симметричности функции $\psi\left(x_{1}, x_{2}, x_{3}, x_{4}\right)$ достаточно рассмотреть функции, представленные в табл. 1, что завершает доказательство леммы 1.

Определим функцию $\Psi$, полагая

$$
\Psi\left(x_{1,1}, x_{1,2}, x_{1,3}, x_{1,4}, \ldots, x_{k, 1}, x_{k, 2}, x_{k, 3}, x_{k, 4}\right)=\psi\left(x_{i, 1}, x_{i, 2}, x_{i, 3}, x_{i, 4}\right),
$$

если все $x_{j, s}$ равны нулю при $i \neq j$. На остальных наборах функция $\Psi$ не определена.

Лемма 2. Функичия $\Psi\left(x_{1,1}, x_{1,2}, x_{1,3}, x_{1,4}, \ldots, x_{k, 1}, x_{k, 2}, x_{k, 3}, x_{k, 4}\right)$ является универсальной функиией $4 k$ переменных.

Доказательство. Рассмотрим произвольную линейную функцию $g$, зависящую от $4 k$ переменных

$$
\begin{aligned}
& g=\beta_{1,1} x_{1,1}+\beta_{2,2} x_{1,2}+\beta_{1,3} x_{1,3}+\beta_{1,4} x_{1,4}+\cdots \\
& \quad+\beta_{k, 1} x_{k, 1}+\beta_{k, 2} x_{k, 2}+\beta_{k, 3} x_{k, 3}+\beta_{k, 4} x_{k, 4}+\gamma
\end{aligned}
$$


Таблица 1.

\begin{tabular}{clll}
\hline \hline & $\psi(0,0,0,0)=0$ & $\psi(0,1,1,1)=0$ & $\psi(1,0,1,1)=0$ \\
0 & $\psi(1,1,0,1)=0$ & $\psi(1,1,1,0)=0$ & \\
\hline$x_{1}$ & $\psi(0,0,0,0)=0$ & $\psi(1,0,0,0)=1$ & $\psi(0,1,1,1)=0$ \\
& $\psi(1,0,1,0)=1$ & $\psi(1,0,0,1)=1$ & \\
\hline & $\psi(0,0,0,0)=0$ & $\psi(1,0,0,0)=1$ & $\psi(0,1,0,0)=1$ \\
$x_{1} \oplus x_{2}$ & $\psi(1,0,1,0)=1$ & $\psi(1,0,0,1)=1$ & \\
\hline & $\psi(0,0,0,0)=0$ & $\psi(1,0,0,0)=1$ & $\psi(0,1,0,0)=1$ \\
$x_{1} \oplus x_{2} \oplus x_{3}$ & $\psi(0,0,1,0)=1$ & $\psi(1,0,0,1)=1$ & \\
\hline & $\psi(0,0,0,0)=0$ & $\psi(1,0,0,0)=1$ & $\psi(0,1,0,0)=1$ \\
$x_{1} \oplus x_{2} \oplus x_{3} \oplus x_{4}$ & $\psi(0,0,1,0)=1$ & $\psi(0,0,0,1)=1$ & \\
\hline & $\psi(1,0,1,0)=1$ & $\psi(1,0,0,0)=1$ & $\psi(0,1,0,0)=1$ \\
& $\psi(0,0,1,0)=1$ & $\psi(0,0,0,1)=1$ & \\
\hline & $\psi(0,1,0,0)=1$ & $\psi(0,0,1,0)=1$ & $\psi(0,0,0,1)=1$ \\
& $\psi(0,1,1,0)=1$ & $\psi(1,0,1,1)=0$ & \\
\hline & $\psi(0,0,1,0)=1$ & $\psi(0,0,0,1)=1$ & $\psi(0,0,1,1)=1$ \\
$x_{1} \oplus 1$ & $\psi(0,1,1,1)=0$ & $\psi(1,0,1,1)=0$ & \\
\hline & $\psi(0,0,0,1)=1$ & $\psi(1,1,0,0)=1$ & $\psi(1,0,1,0)=1$ \\
$x_{1} \oplus x_{2} \oplus 1$ & $\psi(0,1,1,0)=1$ & $\psi(1,1,1,0)=0$ & \\
\hline & $\psi(1,1,0,0)=1$ & $\psi(1,0,1,0)=1$ & $\psi(0,1,1,0)=1$ \\
$x_{1} \oplus x_{2} \oplus x_{3} \oplus 1$ & $\psi(1,0,0,1)=1$ & $\psi(0,1,1,1)=0$ & \\
\hline & & &
\end{tabular}

Если все $x_{j, s}$ равны нулю при $j \neq i$, то функция $g$ равна $\beta_{i, 1} x_{i, 1}+\beta_{i, 2} x_{i, 2}+\beta_{i, 3} x_{i, 3}+$ $\beta_{i, 4} x_{i, 4}+\gamma$. Применив лемму 1 , мы можем подобрать такие пять наборов на соответствующем подкубе размера четыре, что функция $g$ будет единственной линейной функцией, совпадающей с $\Psi$. Проделав эту процедуру для всех $i$, получим множество наборов функции $\Psi$, однозначно задающих $g$ среди среди всех линейных функций. Применив теорему Кронекера-Капелли (см., например, [2], с. 96, 137), избавимся от лишних наборов.

Лемма 3. Пусть $g\left(x_{1}, \ldots, x_{n}\right)-$ универсальная функция $n$ переменных. Пусть функция $f\left(x_{1}, \ldots, x_{n+1}\right)$ совпадает с $g$ при $x_{n+1}=0$ и определена на произвольном квадрате при $x_{n+1}=1$ нелинейным образом. Тогда $f\left(x_{1}, \ldots, x_{n+1}\right)-$ универсальная функиия $n+1$ переменной.

Доказательство. Значения при $x_{n+1}=0$ в силу условий леммы определяют произвольную линейную функцию с точностью до слагаемого $\alpha_{n+1} x_{n+1}$. Поскольку нелинейная функция на квадрате не совпадает ни с одной линейной, то как для функции, так и для ее отрицания найдется набор с координатой $x_{n+1}=1$, на котором они совпадут.

Теорема 1. При $n \geqslant 4$ найдется универсальная функция, заданная на $3,5 n+1+$ 0,5 $(n \bmod 4)$ наборах.

Доказательство. Достаточно применить лемму 2 для наибольшего целого, кратного четырем и не превосходящего $n$. После этого применим лемму 3 необходимое число раз.

Лемма 4. Если функция $n$ переменных равна одной из констант менее, чем на $n+1$ наборе, то она не является универсальной.

Доказательство. Утверждение леммы вытекает из того, что возникающая совместная система на коэффициенты линейной функции будет иметь больше переменных, чем уравнений. 
Таблица 2.

\begin{tabular}{cc}
\hline \hline 0 & $\varphi\left(e_{1}\right)=\varphi\left(e_{2}\right)=\varphi\left(e_{3}\right)=0$, \\
$\varphi\left(e_{4}\right)=\varphi\left(e_{5}\right)=0$ \\
& $\varphi\left(e_{2}\right)=\varphi\left(e_{3}\right)=\varphi\left(e_{4}\right)=0$, \\
$\varphi\left(e_{5}\right)=0, \varphi\left(e_{1}+e_{2}\right)=1$ \\
\hline$x_{1}$ & $\varphi\left(e_{3}\right)=\varphi\left(e_{4}\right)=\varphi\left(e_{5}\right)=0$, \\
& $\varphi\left(e_{5}+e_{1}\right)=\varphi\left(e_{2}+e_{3}\right)=1$ \\
\hline$x_{1}+x_{2}$ & $\varphi\left(e_{2}\right)=\varphi\left(e_{4}\right)=\varphi\left(e_{5}\right)=0$, \\
& $\varphi\left(e_{1}+e_{2}\right)=\varphi\left(e_{2}+e_{3}\right)=1$ \\
\hline$x_{1}+x_{3}$ & $\varphi\left(e_{4}\right)=\varphi\left(e_{5}\right)=0, \varphi\left(e_{5}+e_{1}\right)=1$, \\
& $\varphi\left(e_{3}+e_{4}\right)=\varphi(1)=1$ \\
\hline$x_{1}+x_{2}+x_{3}$ & $\varphi\left(e_{3}\right)=\varphi\left(e_{5}\right)=0, \varphi\left(e_{5}+e_{1}\right)=1$, \\
& $\varphi\left(e_{3}+e_{4}\right)=\varphi\left(e_{2}+e_{3}\right)=1$ \\
\hline$x_{1}+x_{2}+x_{4}$ & $\varphi\left(e_{5}\right)=\varphi\left(e_{1}+e_{3}\right)=\varphi\left(e_{2}+e_{4}\right)=0$, \\
& $\varphi\left(e_{4}+e_{5}\right)=\varphi\left(e_{5}+e_{1}\right)=1$ \\
\hline$x_{1}+x_{2}+x_{3}+x_{4}$ & $\varphi\left(e_{1}+e_{3}\right)=\varphi\left(e_{2}+e_{4}\right)=\varphi\left(e_{3}+e_{5}\right)=0$, \\
& $\varphi\left(e_{4}+e_{1}\right)=0, \varphi(1)=1$ \\
\hline$\left.x_{1}+x_{2}+x_{3}+x_{4}+x_{5}\right)=0$, \\
\hline \hline
\end{tabular}

Лемма 5. При $n \leqslant 3$ не существует универсальных функиий $n$ переменньхх.

Доказательство. Достаточно использовать перебор с применением леммы 4.

Следующая теорема вытекает из теоремы 1 и лемм 4 и 5.

Теорема 2. При $n \geqslant 4$ существует универсальная функция, заданная не более чем на $3,5 n+2,5$ наборах. При $n \leqslant 3$ не существует универсальных функиий. При $n \geqslant 4$ любая универсальная функция п переменных должна быть задана не менее чем на $2 n+2$ наборах.

Замечание 1. В работах [2] и [3] рассматривалась близкая задача доказательства повторности булевой функции.

Верхнюю оценку теоремы 2 можно улучшить, если рассмотреть вместо функции $\psi\left(x_{1}, x_{2}, x_{3}, x_{4}\right)$ частичную функцию $\varphi\left(x_{1}, x_{2}, x_{3}, x_{4}, x_{5}\right)$, определяемую следующими соотношениями. Через $e_{i}$ обозначен соответствующий единичный вектор. Положим $\varphi(0)=0, \varphi(1)=1$. Для любого $i$ положим $\varphi\left(e_{i}\right)=0$. Также положим

$$
\varphi\left(e_{1}+e_{2}\right)=\varphi\left(e_{2}+e_{3}\right)=\varphi\left(e_{3}+e_{4}\right)=\varphi\left(e_{4}+e_{5}\right)=\varphi\left(e_{5}+e_{1}\right)=1
$$

И

$$
\varphi\left(e_{1}+e_{3}\right)=\varphi\left(e_{2}+e_{4}\right)=\varphi\left(e_{3}+e_{5}\right)=\varphi\left(e_{4}+e_{1}\right)=\varphi\left(e_{5}+e_{2}\right)=0 .
$$

На наборах веса три и четыре функция $\varphi\left(x_{1}, x_{2}, x_{3}, x_{4}, x_{5}\right)$ не определена.

Лемма 6. Функиия $\varphi\left(x_{1}, x_{2}, x_{3}, x_{4}, x_{5}\right)$ является универсальной функцией пяти переменHblx.

Доказательство. В силу симметричности функции $\varphi\left(x_{1}, x_{2}, x_{3}, x_{4}, x_{5}\right)$ достаточно рассмотреть функции, представленные в таблицах ниже. Для функций со свободным членом, равным нулю, используем значение $f(0)=0$. Остальные пять значений приведены в табл. 2.

Шестерка значений для функций со свободным членом, равным единице, приведена в табл. 3 . 
Таблица 3.

\begin{tabular}{|c|c|}
\hline 1 & $\begin{array}{c}\varphi\left(e_{1}+e_{2}\right)=\varphi\left(e_{2}+e_{3}\right)=\varphi\left(e_{3}+e_{4}\right)=1 \\
\varphi\left(e_{4}+e_{5}\right)=\varphi\left(e_{5}+e_{1}\right)=\varphi(1)=1\end{array}$ \\
\hline$x_{1}+1$ & $\begin{array}{c}\varphi\left(e_{1}\right)=\varphi\left(e_{1}+e_{3}\right)=\varphi\left(e_{4}+e_{1}\right)=0 \\
\varphi\left(e_{2}+e_{3}\right)=\varphi\left(e_{3}+e_{4}\right)=\varphi\left(e_{4}+e_{5}\right)=1\end{array}$ \\
\hline$x_{1}+x_{2}+1$ & $\begin{array}{c}\varphi\left(e_{1}\right)=\varphi\left(e_{2}\right)=\varphi\left(e_{1}+e_{3}\right)=0 \\
\varphi\left(e_{2}+e_{4}\right)=\varphi\left(e_{5}+e_{2}\right)=0, \varphi\left(e_{1}+e_{2}\right)=1\end{array}$ \\
\hline$x_{1}+x_{3}+1$ & $\begin{array}{c}\varphi\left(e_{1}\right)=\varphi\left(e_{3}\right)=\varphi\left(e_{4}+e_{1}\right)=0 \\
\varphi\left(e_{3}+e_{5}\right)=0, \varphi\left(e_{4}+e_{5}\right)=\varphi(1)=1\end{array}$ \\
\hline$x_{1}+x_{2}+x_{3}+1$ & $\begin{array}{c}\varphi\left(e_{1}\right)=\varphi\left(e_{2}\right)=\varphi\left(e_{3}\right)=0 \\
\varphi\left(e_{4}+e_{1}\right)=\varphi\left(e_{3}+e_{5}\right)=0, \varphi\left(e_{1}+e_{2}\right)=1\end{array}$ \\
\hline$x_{1}+x_{2}+x_{4}+1$ & $\begin{array}{c}\varphi\left(e_{1}\right)=\varphi\left(e_{2}\right)=\varphi\left(e_{4}\right)=0 \\
\varphi\left(e_{1}+e_{3}\right)=\varphi\left(e_{5}+e_{2}\right)=0, \varphi\left(e_{1}+e_{2}\right)=1\end{array}$ \\
\hline$x_{1}+x_{2}+x_{3}+x_{4}+1$ & $\begin{array}{c}\varphi\left(e_{1}\right)=\varphi\left(e_{2}\right)=\varphi\left(e_{3}\right)=0 \\
\varphi\left(e_{4}\right)=\varphi\left(e_{3}+e_{5}\right)=0, \varphi\left(e_{1}+e_{2}\right)=1\end{array}$ \\
\hline$x_{1}+x_{2}+x_{3}+x_{4}+x_{5}+1$ & $\begin{array}{c}\varphi\left(e_{1}\right)=\varphi\left(e_{2}\right)=\varphi\left(e_{3}\right)=0 \\
\varphi\left(e_{4}\right)=\varphi\left(e_{5}\right)=0, \varphi\left(e_{1}+e_{2}\right)=1\end{array}$ \\
\hline
\end{tabular}

Положим

$\Phi\left(x_{1,1}, x_{1,2}, x_{1,3}, x_{1,4}, x_{1,5}, \ldots, x_{k, 1}, x_{k, 2}, x_{k, 3}, x_{k, 4}, x_{k, 5}\right)=\varphi\left(x_{i, 1}, x_{i, 2}, x_{i, 3}, x_{i, 4}, x_{1,5}\right)$,

если все $x_{j, s}$ равны нулю при $i \neq j$. На остальных наборах функция $\Phi$ не определена.

Лемма 7. Функция $\Phi\left(x_{1,1}, x_{1,2}, x_{1,3}, x_{1,4}, x_{1,5} \ldots, x_{k, 1}, x_{k, 2}, x_{k, 3}, x_{k, 4}, x_{k, 5}\right)$ является универсальной функичей $5 k$ переменных.

Доказательство леммы 7 аналогично доказательству леммы 2.

Из лемм 3, 6 и 7 вытекает следующее утверждение.

Теорема 3. При $n \geqslant 5$ существует универсальная функиия, заданная на не более чем $3,2 n+1+0,8(n \bmod 5) \leqslant 3,2 n+4,2$ наборах.

\section{Список литературы}

1. Яблонский С. В., Введение в дискретную математику. Высшая школа, Москва, 2001.

2. Ильин В. А., Ким Г. Д., Линейная алгебра и аналитическая геометрия. МГУ, Москва, 1998.

3. Вороненко А. А., Федорова В. С., Чистиков Д. В., Повторность функций в элементарном базисе. Известия вузов. Математика (2011) №11, 72-77.

4. Вороненко А. А., О сложности доказательства повторности булевых функций в бинарном базисе. Прикладная дискретная математика (2011) 13, №3, 12-16.

Статья поступила 23.03.2012. 